\title{
Das Projekt Interkulturanstalten
}

\section{Die Transformation einer Refugees-Welcome-Initiative in einen kulturellen Begegnungsort}

\author{
Sebastian Beck
}

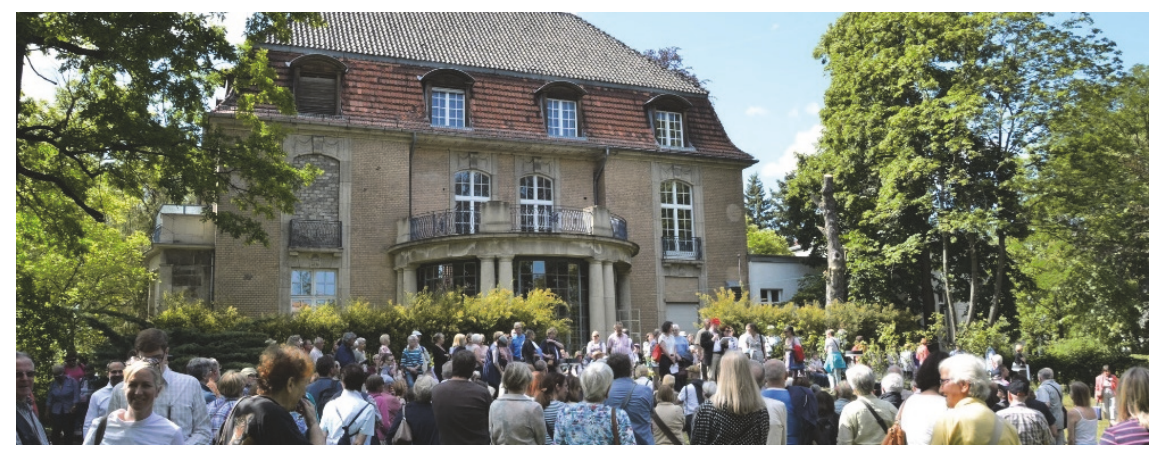

Abbildung 1: Die Interkulturanstalten in der Ulmenallee 35 im Berliner Westend

Foto: B. Arndt (Interkulturanstalten e.V.)

2015/2016 sind viele neue Refugees-Welcome-Initiativen, Unterstützer*innen- und Helfer*innenkreise entstanden, die sich im Kontext der Ersthilfe und der Integration von Geflüchteten engagiert haben. In Bezug auf die Integration von Geflüchteten und die Kooperation von Verwaltung, Politik und Zivilgesellschaft haben diese Initiativen über das Engagement ihrer aktiven Mitglieder deutschlandweit unterschiedlichste Impulse setzen können (vgl. Schiffauer et al. 2017). In vielerlei Hinsicht knüpft dieses Engagement an klassische Aufgaben der Stadtentwicklung an, wie etwa die Wohnraumversorgung oder die Nutzung von Immobilien und Freiflächen für Integrationsangebote. Aus der Perspektive der Stadtforschung lassen sich diese Initiativen als neue intermediäre Akteur*innen in der Stadtentwicklung betrachten (vgl. Beck et al. 2017). Zwar war die Kooperation mit Verwaltung, Politik und Nachbarschaften nie konfliktfrei (vgl. Michels 2017); jedoch konnten in dieser Ausnahmesituation neue Kooperationsformen zwischen Intermediären, Verwaltung und Politik entstehen (vgl. Gesemann 2017). Heute, einige Jahre später, stellt sich die Frage, was in der Folge aus diesen Netzwerken und diesem Engagement geworden ist? 
Sicher, mit der Zeit haben sich die Rahmenbedingungen geändert. Der Zustrom an Geflüchteten hat sich deutlich reduziert, insbesondere im Kontext des Türkei-Deals der deutschen Bundesregierung. Die Lage ist heute weniger dramatisch als zu Beginn, als Angela Merkel noch appellierte: „Wir schaffen das!“ Es geht nun vielmehr um die längerfristige Integration der Geflüchteten und teils auch um eine (Weiter-)Entwicklung urbaner Nachbarschaften. Das verändert zusehends den Kontext, in dem sich die Refugees-Welcome-Initiativen von 2015/2016 heute bewegen. Wie begegnen sie diesen veränderten Rahmenbedingungen? Und welchem Wandel unterliegt das Engagement derjenigen, die in diesen Initiativen aktiv sind? In diesem Beitrag wird dieser Transformationsprozess exemplarisch am Beispiel eines Projekts im Berliner Westend illustriert, dem Projekt Interkulturanstalten e. V. in der Ulmenallee 35 in Berlin.

\section{1 „Mittler, Macher, Protestierer“}

Die Interkulturanstalten stehen paradigmatisch für eine Gruppe von Stadtentwicklungsakteur*innen, die sich als Intermediäre in der Stadtentwicklung bezeichnen lassen. Diese „Mittler, Macher [und] Protestierer“ (Beck \& Schnur 2016) bewegen sich in einem Spannungsfeld zwischen Government und Governance, bei dem es nicht nur um die Frage geht, wer mit welcher Legitimität welche Entscheidungen treffen kann, sondern vor allem auch um die Frage, wer mit welchen Kompetenzen und Ressourcen Projekte anstößt und umsetzt. Diese Debatte um Intermediarität ist keineswegs neu, aber aufgrund veränderter Kontexte aktueller denn je, weil sich die Art und Weise ändert, in der Intermediäre auftreten: nicht nur als Vermittelnde, sondern auch als Impulsgebende bzw. als Umsetzende konkreter lokaler Projekte entlang formeller, aber insbesondere eben auch entlang informeller Schnittstellen und Netzwerkstrukturen. Entsprechend lassen sich Intermediäre zeitgemäß definieren als „Vermittler zwischen Politik/Verwaltung, Wirtschaft und Bürgerinnen und Bürgern, und/oder aktive Koproduzenten von Stadt in variierenden Akteursnetzwerken" (Beck \& Schnur 2016: 15). Das Feld der Intermediären befindet sich im Umbruch. Typologisch ließen sich dabei unterscheiden (vgl. Abb. 2): klassische Intermediäre (z. B. Parteien, Gewerkschaften), Neo-Intermediäre (z. B. soziale Bewegungen, Social-Media-basierte Initiativen) und Para-Intermediäre (z. B. kommerzielle Urban Labs oder Think Tanks).

Mit den Neo-Intermediären ist im Kontext von Postmoderne und Netzwerkgesellschaft ein Akteurstypus entstanden, der jenseits finanzstarker, etablierter oder mitgliederstarker Verbandsstrukturen (klassische Intermediäre, s. u.) in Form flexibler Netzwerke praktisch, anlassbezogen und zivilgesellschaftlich orientiert agiert. Über Social Media, Communitys und Follower, ihre Anbindung an die modernen, kreativen Milieus und deren Lebenswelten, über ein Gespür für Konflikte, 
Herausforderungen und Entwicklungschancen sind sie in der Lage, Lösungs- und Handlungsansätze zu entwickeln und diese als netzwerkartige Akteurskoproduktionen in der Stadt umzusetzen.

Para-Intermediäre sind deutlich stärker mit dem marktwirtschaftlichen Sektor verbunden. Als Immobilienverband, Think Tank, Stiftung oder auch als diskursorientiertes Urban Lab ist mal mehr, mal weniger transparent, dass sie sich in den Aushandlungsprozessen der Stadtentwicklung als Shareholder bewegen und die Zivilgesellschaft eher aus der Perspektive von Marktpotenzialen heraus betrachten. Auch wenn ihr Handeln manchmal dialogorientiert erscheint, unterliegen ihre tatsächlichen Ziele eher einer kapitalorientierten Renditelogik als einer stadtgesellschaftlichen Gemeinwohlorientierung.

Die klassischen Intermediären stehen angesichts der Modernisierung und Dynamisierung des intermediären Feldes unter Transformationsdruck. Wo in der korporatistischen Nachkriegsmoderne „Big Government“, „Big Business“ und „Big Labour“ die wesentlichen Eckpunkte des Gemeinwohls aushandelten, sind die einst stabilen gesellschaftlichen Bindungen und Verankerungen dieser Akteur*innen (Gewerkschaften, Parteien, karitative Einrichtungen etc.) zunehmend brüchig geworden. Sie versuchen momentan, sich von den einstigen vertikalen Mittler*innen zu ebenfalls horizontal agierenden Governance-Akteur*innen weiterzuentwickeln.

\section{Klassische Intermediäre Neo-Intermediäre $\quad$ Para-Intermediäre}
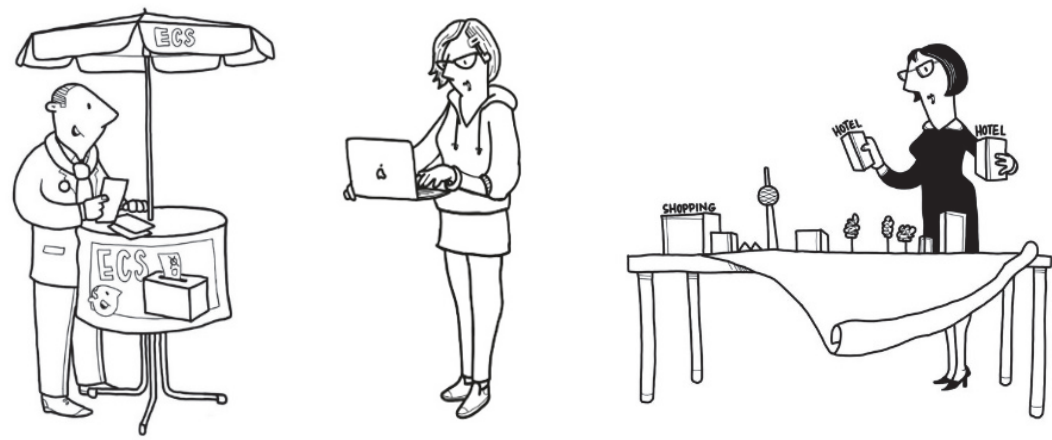

Abbildung 2: $\quad$ Klassische Intermediäre, Neo-Intermediäre und Para-Intermediäre Quelle: C. Kellner// studio animanova.

Die intermediären Akteur*innen - und insbesondere die Neo-Intermediären - stehen paradigmatisch für einen Wandel von etablierten Government-Strukturen hin 
zu einer Urban Governance. In diesem Wandlungsprozess, der das Handeln, die Rollen und die Legitimitäten der Gesamtheit der Stadtentwicklungsakteur*innen betrifft, scheinen die Intermediären von besonderer Bedeutung zu sein, weil es quasi in ihrer DNA liegt, „Zwischen“ Kommunen und Einzelpersonen wie auch „Zwischen“ den vertikalen Strukturen von Government und den horizontalen Kooperationen in Governance-Prozessen zu agieren. Unabhängig von der Frage, ob die Intermediären die Produkte oder die Treiber dieses Wandlungsprozesses sind, bleibt doch zu konstatieren, dass sie zu immer engeren wechselseitigen Bezügen von Government- und Governance-Strukturen bzw. zu einer Konvergenz dieser beiden Arenen beitragen (vgl. Abb. 3).
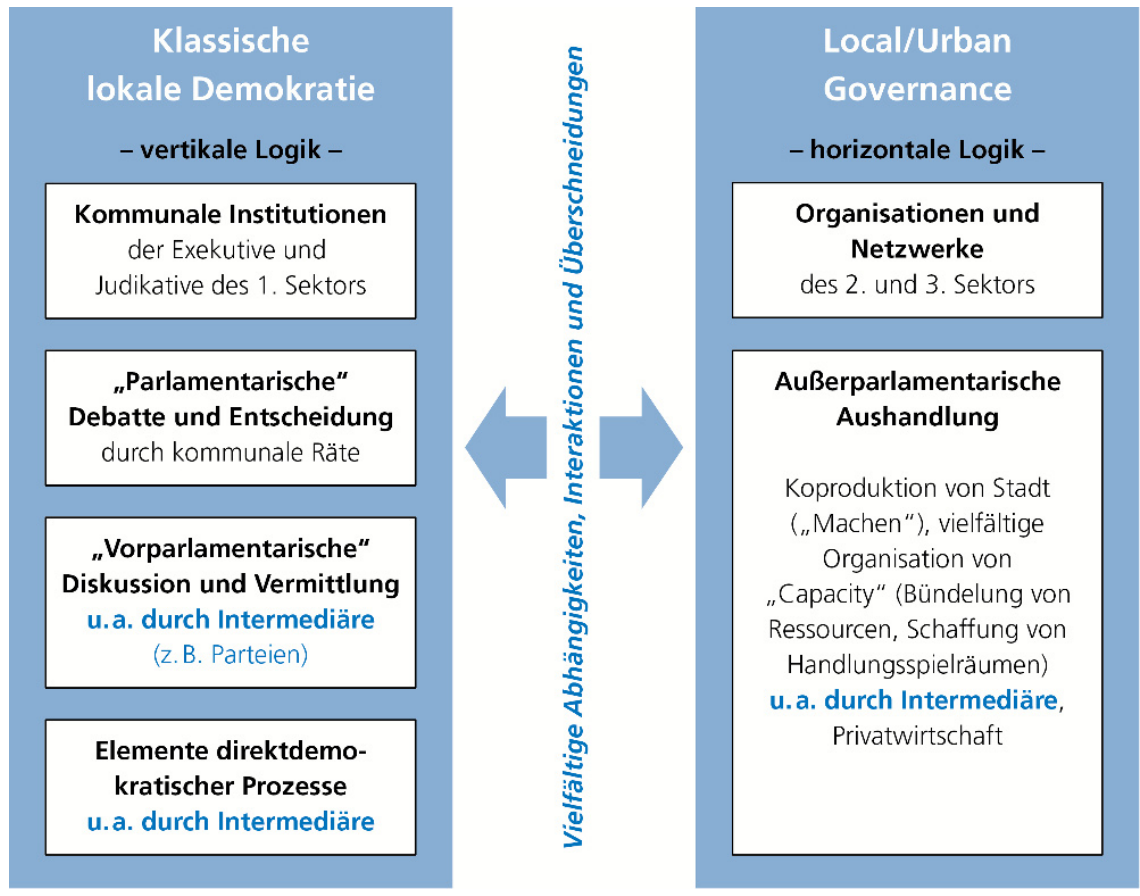

Abbildung 3: $\quad$ Intermediäre in den Arenen der klassischen lokalen Demokratie und der Local/Urban Governance

Quelle: Beck \& Schnur (2016: 21).

Es wäre verkürzt, die Intermediären auf eine bipolare Vermittlungsperspektive zwischen Bürger*innen und der Kommune oder dem Staat zu reduzieren. Sie sind 
Bestandteil von Aushandlungsprozessen, an denen auch der wirtschaftliche Sektor beteiligt ist (vgl. Maikämper 2016). Sie knüpfen an Bezüge zur Bürgergesellschaft an, die etwa Heinze \& Olk (2001) als ,die intermediäre Sphäre zwischen Staat, Marktwirtschaft und dem informellen Bereich der Privathaushalte" beschrieben hatten. Die Intermediären sind ein Bestandteil komplexer trisektoraler Aushandlungsprozesse zwischen Verwaltung und Politik, (lokaler) Wirtschaft und einzelnen Bürger*innen (vgl. Abb. 4). Sie agieren mal mehr als Mittler*innen, mal als Macher*innen oder eben auch als Protestierer*innen, je nachdem, in welcher Konstellation sie sich in Bezug auf ihr aktuelles Vorhaben mehr Vorteile versprechen können. Dabei verfügen sie in der Regel über benennbare Kompetenzen und Ressourcen, die sich entlang der Begrifflichkeiten von Pierre Bourdieu in Bezug auf ökonomisches, soziales und kulturelles Kapital beschreiben lassen (vgl. Beck \& Schnur 2016: 67 ff.). Damit lassen sich die Intermediären auch als urbane Ressource verstehen, die im Sinne eines „Capacity Buildings“ die Handlungsmöglichkeiten der Stadtentwicklung potenziell erweitert.

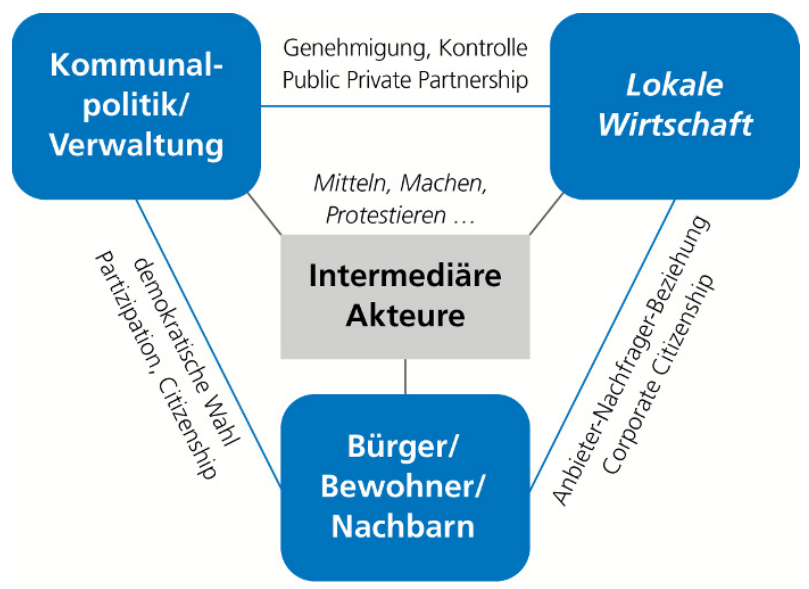

Abbildung 4: Intermediäre im Kräftedreieck von Kommunalpolitik/-verwaltung, lokaler Wirtschaft und Bürgergesellschaft

Quelle: Beck \& Schnur (2016: 23).

Die Intermediären sind allerdings keineswegs ein Vademecum „richtiger“ Stadtentwicklungspolitik, sondern vielmehr die „Spitze des Eisbergs“ in einem zusehends vielfältigeren und immer stärker vernetzten „Akteursaquarium“. Sie stehen paradigmatisch für eine Entwicklung, in der sich Government- und GovernanceStrukturen miteinander vernetzen und weiterentwickeln. Dabei stellt sich die 
Frage, ob Intermediäre über die Akteursgruppe der Intermediären hinaus für eine bestimmte Haltung stehen, Stadtentwicklungsprozesse voranzutreiben, für so etwas wie Intermediarität bzw. eine ,intermediäre Kultur“ (ebd.: 58).

\section{Refugees-Welcome-Initiativen als Neo-Intermediäre}

Eine empirische Untersuchung der Intermediären mit dem Fokus auf Berlin wurde 2016/2017 seitens des vhw Bundesverbands für Wohnen und Stadtentwicklung e. V. in Kooperation mit dem Berliner Institut für Demokratische Entwicklung und Soziale Integration (DESI) und der Berliner L.I.S.T. - Lösungen im Stadtteil Stadtentwicklungsgesellschaft mbH 2016/2017 durchgeführt (vgl. Gesemann et al. 2017). Ziel war es, am Beispiel Berlins die Vielfalt der intermediären Stadtentwicklungsakteur*innen in den Blick zu nehmen und Veränderungen in den Aushandlungsprozessen zwischen Kommune, Wirtschaft, organisierter Zivilgesellschaft und einzelnen Bürger*innen auszuloten. Im Fokus dieser Studie standen insbesondere diejenigen Stadtentwicklungsakteur*innen, die sich als Neo-Intermediäre (vgl. Beck \& Schnur 2016: 45 ff.) bezeichnen lassen. Denn hier sollte es darum gehen, inwieweit sich infolge einer zunehmenden Diversifizierung des Spektrums intermediärer Akteur*innen neuartige Entwicklungspfade erkennen ließen. In der Studie wurden 30 Berliner Stadtentwicklungsinitiativen untersucht, die sich durch neuartige Kooperationsformen, Veränderungen in den diesbezüglichen formellen bzw. informellen Kooperationsstrukturen und Schnittstellen oder eigenständige Impulse zur Stadtentwicklung auszeichnen.

Der Schwerpunkt der hier vorgestellten Studie lag insbesondere auf Initiativen aus den Themenfeldern Wohnen und Willkommenskultur, weil diese im Untersuchungszeitraum in Berlin einer besonderen Entwicklungsdynamik unterlagen: in Bezug auf die Wachstums-, Aufwertungs-, Verdrängungs- und Gentrifizierungsprozesse des Berliner Wohnungsmarkts und eben auch in Bezug auf den starken Bevölkerungszuwachs in Berlin durch die Aufnahme von mehreren Tausend Geflüchteten in Berlin - allein im Jahr 2015 waren dies 80.000 Personen (vgl. Beck et al. 2017: 3). Bereits 2017 ließen sich dabei zwei wesentliche Momente festhalten, die auch für das weitere Engagement von Neo-Intermediären und auch von Refugees-Welcome-Initiativen von zentraler Bedeutung zu sein scheinen:

- Zum einen sehen sich neo-intermediäre Initiativen der Herausforderung gegenübergestellt, ihr Engagement zu verstetigen bzw. ihr Engagement wechselnden Rahmenbedingungen gegenüber anzupassen. In Bezug auf RefugeesWelcome-Initiativen ließ sich dazu bereits 2017 festhalten, dass diese Initiativen den von ihnen bearbeiteten thematischen Radius immer stärker auffächerten. Auf diese Weise entwickelten sich aus reinen Unterstützerinitiativen 
politisch motivierte Akteur*innen, die ihren Handlungsrahmen beständig ausweiteten und über Wohninitiativen für Geflüchtete auch Einfluss auf die städteplanerische Gestaltung nahmen (ebd.: 4).

- Zum anderen entwickeln sich im Kontext des Engagements von neo-intermediären Initiativen neue Kooperationsstrukturen zwischen Zivilgesellschaft und Verwaltung - allein schon aus dem Moment heraus, dass diese Initiativen Dinge vorantreiben, Fragen stellen, Reaktionen einfordern und teilweise bereits sogar die Schaffung neuer Schnittstellen zwischen Verwaltung und Zivilgesellschaft initiieren: „In Berlin sieht sich die lokale Verwaltung nun damit konfrontiert, eine Haltung zu den neuen selbstbestimmten und an Koproduktion orientierten Praktiken des ,Stadtmachens` zu entwickeln“ (ebd.: 10).

\section{Refugees-Welcome-Initiativen als lokale Nachbarschaftsakteur*innen?}

Über die hier dargestellten Prozesse der Verstetigung und des Schaffens neuer Kooperationsstrukturen hinaus lässt sich nun allerdings ein drittes wesentliches Moment in Bezug auf das Engagement von Refugees-Welcome-Initiativen festhalten. Im lokalen Engagement für Geflüchtete können sich Interaktionen zwischen Geflüchteten und der Nachbarschaft, in der das Engagement der Refugees-WelcomeInitiativen stattfindet, ergeben, die wiederum einen Ort haben: die Adresse, die Räumlichkeiten, der städtische Raum, an dem das Engagement von RefugeesWelcome-Initiativen stattfindet. Tatsächlich ließe sich dieses Moment auch für die im Rahmen der hier vorgestellten Studie untersuchten Initiativen festhalten, wie etwa die Interkulturanstalten oder auch das Berliner Projekt Hellersdorf hilft. Beide werden hier kurz steckbriefartig vorgestellt:

Die Initiative Hellersdorf e. V. hilft ist eine seit 2013 bestehende Willkommensinitiative, die sich als Reaktion auf zunehmende rassistische Stimmungen im Umfeld einer Flüchtlingsunterkunft in Berlin-Hellersdorf gegründet hat. Die Initiative versucht, mit antirassistischer Arbeit im Wohnumfeld eine Willkommenskultur zu etablieren, z. B. mit Workshops in angrenzenden Schulen, und macht sich für die gesellschaftliche Partizipation der Geflüchteten im Bezirk stark. Von Beginn an machte die Initiative u. a. auf Probleme bei der inzwischen bundesweit bekannten ehemaligen Betreibergesellschaft der Flüchtlingseinrichtung „PeWoBe“ aufmerksam (vgl. Gesemann et al. 2017). Das lokale Engagement dieser Initiative manifestiert sich u. a. in der Einrichtung „LaLoKa“, einem Treffpunkt für Geflüchtete außerhalb der Flüchtlingsunterkunft, der als Treffpunkt, aber auch als Raum für kleinere Veranstaltungen nutzbar ist.

Die Initiative Interkulturanstalten geht im Kern auf das Engagement der Initiative „Willkommen im Westend“ zurück, die sich ebenfalls bereits im Jahr 2013 gründete. Seit der Gründung der Initiative ist mittlerweile eine dichte Infrastruktur 
zur Unterstützung von Geflüchteten entstanden. Aus dieser gesellschaftlich breit getragenen Initiative ist zudem 2017 das Projekt „Interkulturanstalten“ hervorgegangen, bei dem ein altes Sanatorium zu einer vielfältig genutzten sozialen und kulturellen Einrichtung umgebaut wurde. Die Initiative „Willkommen im Westend" ist bis heute ein fester Ansprechpartner für Politik und Verwaltung und Vertreter*innen der Initiative berichten regelmäßig im Integrationsausschuss des Bezirks. Die Initiative funktioniert zudem als dynamisches Gebilde mit einer sich ständig verändernden personellen Situation (vgl. ebd.). Die Räumlichkeiten der Interkulturanstalten bieten der Initiative seit 2017 einen konkreten Ort, der nicht nur für die Flüchtlingsarbeit genutzt wird, sondern einen offenen Raum darstellt, der bewusst Begegnungen zwischen Geflüchteten und Nachbarschaft ermöglicht und dabei als Ort für Veranstaltungen wie auch als Räumlichkeit für Projekte seitens der Geflüchteten selbst zur Verfügung steht.

Wenn es Geflüchteten-Initiativen gelingt, einen städtischen Raum zu bespielen, vervielfacht sich ihre potenzielle Wirksamkeit: Der zur Verfügung stehende Ort entwickelt eine gewisse „Magie“ und kann im wahrsten Sinne als „Kapital“ wirken: indem er den Initiativen eine „Sichtbarkeit“" verschafft („LaLoKa“, „Interkulturanstalten") und indem er den Initiativen die Möglichkeit verschafft, diesen Raum zu bespielen. Dieser doppelte Verweis auf zwei Projekte wird an dieser Stelle deshalb aufgegriffen, um das Projekt der Interkulturanstalten nicht solitär im Raum stehen zu lassen. Vielmehr ließen sich an dieser Stelle mit Blick auf Berlin (wie etwa auf das Sharehouse Refugio in Neukölln) ober auch mit Blick auf Beispiele aus anderen deutschen Kommunen (wie etwa auf das Grandhotel Cosmopolis in Augsburg) weitere Projekte von, mit und für Geflüchtete(n) aufzeigen, die ebenfalls einen städtischen Raum bespielen, der für öffentliche Veranstaltungen und nachbarschaftliche Begegnungen bzw. Initiativen aufseiten von Geflüchteten genutzt werden kann. Dieser programmierte und bespielte städtische Raum wirkt sich potenziell förderlich auf die Legitimität dieser Initiativen als handelnde stadtentwicklungspolitische Akteur*innen wie auch auf die Akzeptanz von Geflüchteten als Nachbar*innen aus, wie sich am Beispiel der Interkulturanstalten wird zeigen lassen.

Festzuhalten bleibt hier, dass die Rolle von Refugees-Welcome-Initiativen als Nachbarschaftsakteur*innen vielfältige weiter gehende Fragen impliziert, die an dieser Stelle noch nicht abschließend geklärt sind, allem voran in Bezug auf eine genauere Klärung des Begriffs „Nachbarschaftsakteur*in“ oder in Bezug auf den Begriff „Nachbarschaft“. Es sei an dieser Stelle lediglich darauf hingewiesen, dass es in Bezug auf das Engagement von Refugees-Welcome-Initiativen offenbar wichtig ist, dem Ort dieses Engagements eine besondere Aufmerksamkeit zukommen zu lassen und diesen Ort als „lokale Ressource“ in Bezug auf die Wirksamkeit von Refugees-Welcome-Initiativen auf die soziale Kohäsion vor Ort, die Inte- 
gration von Geflüchteten, das Miteinander von Geflüchteten und Nachbarschaften sowie selbstverständlich auch für die Kontakt- und Aufenthaltsqualitäten einer Nachbarschaft selbst zu begreifen.

\section{Die Interkulturanstalten: Eckdaten}

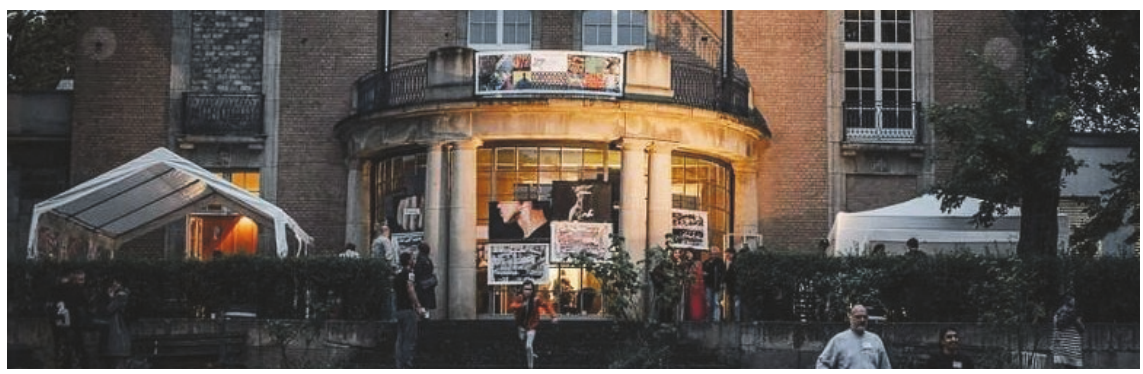

Abbildung 5: $\quad$ Sommerfest der Interkulturanstalten 2017

Foto: zur Verfügung gestellt vom Interkulturanstalten e. V.

Die Interkulturanstalten befinden sich in der Ulmenallee 35 im Berliner Westend in den Räumlichkeiten der 1887 erbauten „Kulturanstalten Westend für Nervenkranke“ (vgl. Interkulturanstalten 2018). Die Initiative beschreibt ihre Räumlichkeiten in der „Ulme 35“ wie folgt:

„Die ULME 35 ist ein Zauberberg, inmitten einer kleinen Parkanlage. Die Gesellschaftsräume in der Beletage - das ehemalige Billardzimmer, die Bibliothek, Musikund Damensalon, ein Hörsaal, ein großer Wintergarten - sind wie geschaffen als Begegnungsort für Geflüchtete und Westender (und alle anderen)“ (ebd.).

Die Immobilie der Interkulturanstalten war 2002 von der Berliner Charité geschlossen worden und stand im Anschluss daran 15 Jahre leer. Das Sanatorium an der Ulmenallee 35 steht heute unter Denkmalschutz und befindet sich im Berliner Westend in einer der ältesten Villenkolonien Berlins. In der Nachbarschaft wurden in den vergangenen Jahren mehrere Flüchtlingsheime eingerichtet, in denen im Winter 2015 insgesamt ca. 3500 Geflüchtete lebten. Heute besteht unweit der Ulme 35 noch eine Unterkunft, in der aktuell ca. 400 Geflüchtete leben. 2017 gelang es aus dem Engagement der Initiative Willkommen im Westend heraus, das Gebäude im Rahmen des Vereins Interkulturanstalten e. V. über Mittel des Integrationsfonds des Bezirks Charlottenburg-Wilmersdorf nutzbar zu machen. Der Verein betreibt die Immobilie und hat sich zum Ziel gesetzt, das nachbarschaftli- 
che Miteinander zwischen Geflüchteten und Nachbarschaft über Dialog, Kunst und Kultur zu fördern. Eine langfristige Finanzierung des Projekts ist noch Work in progress.

Die Präsenz der Interkulturanstalten in einem gutbürgerlichen Berliner Villenviertel war zu Beginn recht kontrovers. In der Nachbarschaft bestand die Befürchtung, dass sich der Immobilienwert der angrenzenden Villen - wenn nicht des gesamten Viertels - mindern könnte, wenn in der Ulmenallee 35 nun dauerhaft ein Begegnungsort für Geflüchtete eingerichtet werden würde. Es bestanden Ängste vor „,dem anderen“, „dem Unbekannten“ und „,dem potenziell Gefährlichen“ und es gab Widerstand gegen das Projekt. Vereinzelt wurden auch Unterschriften gesammelt. Trotzdem gelang es den Aktiven, die politische Unterstützung des Bezirks Charlottenburg-Wilmersdorf für das Projekt Interkulturanstalten zu gewinnen. Die Initiative gründete einen Verein und konnte auf diese Weise Mittel des Integrationsfonds in Anspruch nehmen, mit denen die Immobilie gemietet werden konnte und mit denen sich auch zwei bezahlte Stellen finanzieren ließen. Der unschlagbare Vorteil der Initiative bestand in ihrer starken Verwurzelung mit den Aktivitäten und den Aktiven der Initiative Willkommen im Westend (s. o.), die sich im Bezirk Charlottenburg-Wilmersdorf - an wechselnden Orten und mit vielfältigen Kooperationspartnern - bereits zu einer etablierten integrationspolitischen Akteurin entwickelt hatte. Der nachbarschaftliche Widerstand gegenüber dem Projekt ließ in dem Moment nach, in dem die Interkulturanstalten ihren Betrieb aufnahmen und zunehmend nachbarschaftliche Kontakte mit Geflüchteten stattfinden konnten. Zumindest in einem Einzelfall wurde dabei aus einer Nachbarin, die das Projekt zunächst deutlich kritisiert hatte, eine engagierte Unterstützerin der Interkulturanstalten, die kurzfristig sogar einem geflüchteten syrischen Arzt in ihrer Villa im Westend ein Zimmer zur Verfügung stellte. Eine von Kritiker*innen des Projekts initiierte Anfrage im Bezirksrat zur längerfristigen Perspektive der Interkulturanstalten führte $2018 \mathrm{zu}$ einer Neuauflage des Bebauungsplans, in deren Zuge für das Gelände der Interkulturanstalten allerdings eine kulturelle Nutzung der Immobilie festgeschrieben wurde.

Aktuell (Stand: August 2018) steht die Ulme 35 den Interkulturanstalten im Rahmen einer Zwischennutzung zur Verfügung. Ein langfristiges Nutzungskonzept soll in Kooperation zwischen den (kommunalen) Eigentümern und dem Interkulturanstalten e. V. erarbeitet werden (Interkulturanstalten 2017). Erdgeschoss und Hochparterre wurden in Kooperation mit dem Verein instand gesetzt und werden seit Februar 2017 genutzt. Die Räumlichkeiten sind weitläufig und umfassen einen Hörsaal, ein Café, eine Bibliothek, eine Koch-Werkstatt, einen Seminarraum sowie Mietflächen für Geflüchtetenprojekte und Künstler (Ateliers). 


\section{Die Interkulturanstalten als Intermediäre und Koproduzenten von Nachbarschaft}

Der Verein Interkulturanstalten e. V. ist zwar aus dem Engagement der Initiative Willkommen im Westend hervorgegangen; der Personenkreis der Aktiven ist aber nicht deckungsgleich. Bei den Aktiven von Willkommen im Westend handelt es sich eher um die klassische Klientel der Refugees-Welcome-Bewegung: „Weiblich, gebildet, progressiv, älter" (Interview mit einer Aktiven der Interkulturanstalten e. V. im August 2017). Das Klientel des Interkulturanstalten e. V. ist ,seriöser und spricht ein vielfältigeres Publikum an" (ebd.). Die Schnittstellen mit Politik, Verwaltung und lokaler Wirtschaft sind vielfältig, da sich die Aktiven der beiden Initiativen in Teilen überschneiden. Die Trennung der beiden Initiativen voneinander verläuft von daher relativ diffus und ein Auseinanderdividieren der beiden Gruppen ist auch nicht gewollt. Ganz im Gegenteil: Die bestehenden Kontakte und Kooperationen von Willkommen im Westend ließen sich gewinnbringend für die Arbeit des Interkulturanstalten e. V. nutzen. So wurden die Bestrebungen der Interkulturanstalten, eine politische und finanzielle Lösungsstrategie zur Nutzung der Ulme $35 \mathrm{zu}$ finden, unterstützt.

Die Interkulturanstalten erfüllen klassische intermediäre Funktionen im Kräftedreieck zwischen Verwaltung und Politik, lokaler Wirtschaft und Bürger*innen. Die finanzielle Förderung erfolgt über den Masterplan für Sicherheit und Integration über den Bezirk Charlottenburg-Wilmersdorf und ermöglicht eine Nutzung der Räumlichkeiten der Eigentümer GSE gGmbH und der (kommunalen) BIM. Zudem lassen sich die Interkulturanstalten für die Aktivitäten der Initiative Willkommen im Westend nutzen, die ihrerseits weiterhin über vielfältige Kooperationen mit und Schnittstellen zur Berliner Verwaltung und Politik verfügt (vgl. Gesemann et al. 2017: 55 ff.). Die Arbeit der Interkulturanstalten selbst richtet sich aktiv an Geflüchtete und die Nachbarschaft: „,Der Zweck des Vereins ist die Förderung der Hilfe für politisch, rassisch oder religiös Verfolgte, für Geflüchtete und Vertriebene. In dem Gebäude Ulmenallee 35 entsteht ein Zentrum der Begegnung für Geflüchtete, Nachbarn, Kunst und Zivilgesellschaft" (Interkulturanstalten 2017).

Die Kooperationen der beiden Initiativen mit dem Bezirk lassen sich als sehr gut beschreiben. Die Aktiven sind nicht nur tatsächlich in Kooperationsprozesse zur Arbeit an der Integration von Geflüchteten im Bezirk eingebunden, sondern die Aktiven kennen die zuständigen Ämter und Personen auch persönlich, teilweise bis hin zur gegenseitigen Kenntnis der Mobilfunknummern. Die Initiative Willkommen im Westend verfügt allerdings auch über Kooperationsstränge auf Landesebene, die dagegen weniger gut ausgeprägt und teils konfliktbeladen sind, etwa in Bezug auf die gemeinsame Arbeit an einem landesweiten Masterplan für Integration. Dabei arbeiten die Interkulturanstalten letztlich weniger ,politisch“ 
als die Initiative Willkommen im Westend. Sie sind eher ein Ort für kooperative Impulse und ein Moment der „Balance“ gegenüber der Strategie der „konfrontativen Kooperation" der Refugees-Welcome-Initiative Willkommen in Westend. Die Interkulturanstalten sind mehr Begegnungsraum für die (deutsche) Nachbarschaft und für Geflüchtete. Die Initiative Willkommen im Westend ist durch ihre jahrelange Integrationsarbeit mit Geflüchteten und für Geflüchtete dagegen politisierter: „Der anfangs karitativ orientierten Hilfe folgte eine schnelle Politisierung der Initiative“" (Gesemann et al. 2017: 55). Diese Politisierung bezog sich zunächst auf die strukturellen Defizite, die Intransparenz und Bürokratie im Umgang der Verwaltung mit den Geflüchteten (ebd.). In der Auseinandersetzung mit diesen Problematiken entwickelte die Initiative schließlich viele eigene Kompetenzen, insbesondere auch im Themenfeld des Umgangs mit minderjährigen, unbegleiteten Geflüchteten, und entwickelte eigenständige politische Lösungsvorschläge.

Die Interkulturanstalten sind vor allem aber auch Koproduzenten von Nachbarschaft. Mit dieser Orientierung bietet die Initiative für die Refugees-WelcomeInitiative Willkommen im Westend einen neuen Entwicklungspfad in Richtung Nachbarschaftsakteurin: weniger „politisch“, mit einem über den „Kern“ der Refugees-Welcome-Bewegung hinausgehenden Kreis von Aktiven und mit einer im Ergebnis relativ breiten (nachbarschaftlichen und geflüchtetenspezifischen) Zielgruppenorientierung. Das Angebot der Interkulturanstalten beinhaltet im Kern ein Sprachcafé (mittwochs) sowie einen regulären Cafébetrieb im Sinne eines Nachbarschaftstreffpunkts (mittwochs bis sonntags) und häufig findet freitags eine Kinovorführung statt. Das aktuelle Programm ist einsehbar auf www.interkulturanstalten.de. Zudem haben die Interkulturanstalten vielfältige Mieter*innen, etwa die Sozialberatung Mada e. V., die syrische Frauenzeitschrift Saiedat Souria oder ein Drucker-Atelier. Die Interkulturanstalten wirken als Ort mit Räumlichkeiten, Personal und Mieter*innen in die Nachbarschaft (über Kooperationen mit Kirchen, Wohnheimen und Schulen), in den Bereich Kunst und Kultur (etwa über die Senatskanzlei für kulturelle Angelegenheiten oder die Universität der Künste oder die Komische Oper Berlin), in den Bereich der Beratung und Begegnung (etwa über die Kooperation mit dem AWO Jugend-Migrationsdienst) und in die Arbeitsvermittlung (etwa über die Arbeitsagentur oder die Handwerkskammer). ${ }^{1}$

\section{$6 \quad$ Was bleibt?}

In Bezug auf die Versteigung der Aktivitäten von lokalen Refugees-Welcome-Initiativen lassen sich die Interkulturanstalten zunächst als „Best-Practice“-Modell begreifen, weil es ihnen gelungen ist, das Engagement für Geflüchtete und mit

1 Weitere Details: siehe Interkulturanstalten 2017: $2 \mathrm{ff}$. 
Geflüchteten in einen neuen Kontext zu stellen: von der Ersthilfe und der Unterstützung der zuständigen Stellen bei der Integration vieler Tausender Geflüchteter hin zu einem Expertisenetzwerk bei der lokalen Integration von Geflüchteten und zu einem Nachbarschaftsort, an dem sich Geflüchtete und Nicht-Geflüchtete gemeinsam für ein wertschätzendes Miteinander engagieren und gemeinsam attraktive Projekte in den Bereichen Dialog, Kunst und Kultur gestalten können. In der Weiterentwicklung von einer Akteurin der Flüchtlingshilfe (Willkommen im Westend) zu einer „Nachbarschaftsakteurin“ (Interkulturanstalten e. V.) ist es hier einer Willkommensinitiative gelungen, ihr Engagement als Expert*innen bei der Integration von Geflüchteten aufrechtzuerhalten und sich gleichzeitig ein neues Tätigkeitsfeld zu erschließen. Erstaunlicherweise war es möglich, die politische und die finanzielle Unterstützung zu organisieren, um in diesem Sinne eine leer stehende Gründerzeitvilla für das Vorhaben der Initiative Interkulturanstalten zu gewinnen, Nachbarschaft gemeinsam mit Geflüchteten und Nicht-Geflüchteten zu gestalten und zu neuem Denken, zu gelebter Willkommenskultur, anzuregen. Dabei mussten auch vor Ort, in der direkten Nachbarschaft, Distanzen zu und Konflikte gegenüber einem solchen Projekt der Geflüchtetenarbeit überbrückt werden, was offensichtlich bislang zu gelingen scheint. Dabei wird deutlich, dass der Ort selbst, in diesem Fall die Gründerzeitvilla in der Ulmenallee 35, eine Ressource ist, die intermediäres und nachbarschaftliches Handeln massiv unterstützt und als „lokales Kapital“ wirken kann. Die Immobilie Ulme 35 ermöglicht vielfältige Aktivitäten und Projekte, die sich für die Geflüchtetenarbeit, aber auch einfach nur für gelebte Nachbarschaft nutzen lassen.

Die Interkulturanstalten erfüllen eine klassische intermediäre Funktion in der Vermittlung zwischen Politik/Verwaltung, lokaler Wirtschaft, organisierter Zivilgesellschaft, Bürger*innen und der Nachbarschaft bzw. den Nachbarschaften der betriebenen Immobilie Ulme 35. In Kooperation mit der Initiative Willkommen im Westend können sie ein breites inhaltliches Spektrum und ein unterschiedliche Zielgruppen ansprechendes Gesamtpaket an Aktivitäten anbieten, das kurz- und mittelfristig eine Kontinuität und Verstetigung der Initiativenarbeit ermöglicht - auch und gerade vor dem Hintergrund geänderter Rahmenbedingungen der Situation von Geflüchteten in Deutschland. Dieses von den Interkulturanstalten ermöglichte und initiierte Gesamtpaket an Aktivitäten verbindet interessanterweise unterschiedliche Stränge lokaler Demokratie. Die Interkulturanstalten interagieren mit den Repräsentant*innen der repräsentativen Demokratie und beteiligen sich gleichzeitig an dialogorientierten, deliberativen Beteiligungsformen (in der Aushandlung der Mittel für den Betrieb der Immobilie, in der Beteiligung an flüchtlingspolitischen Netzwerken oder auch in der Beteiligung an flüchtlingspolitischen Debatten auf Bezirks- und Landesebene). Sie setzen sich mit Formen direkter Demokratie auseinander (in der Auseinandersetzung mit gegen das Projekt gerichteten Unterschriftenaktionen aus 
der Nachbarschaft), protestieren beizeiten (etwa gegen die flüchtlingspolitische Debatte auf Landes- oder Bundesebene) bzw. engagieren sich im Sinne einer Bürgerinitiative (zur Nutzung der bislang ungenutzten Immobilie Ulme 35). Oder sie treten als eine Variante einer sozialen Bewegung auf, die sich für ein vielfältiges interkulturelles Miteinander einsetzt (Förderung der Begegnung von und des Miteinanders von Geflüchteten und Nachbarschaft). Ebenso sind sie ganz gewiss ein Ort des bürgerschaftlichen Engagements (Vorantreiben diverser politischer, kultureller und integrationsbezogener Projekte und Aktionen). Roland Roth (2015) hat diese unterschiedlichen politischen Arenen als nebeneinander und miteinander in Verbindung stehende Elemente einer vielfältigen Demokratie beschrieben und die intermediären Akteur*innen als potenzielle Multiplikator*innen der Leitidee einer solchen vielfältigen Demokratie benannt. Politik zu machen, bedeutet eben nicht nur, sich im repräsentativen politischen System zu bewegen, sondern es bedeutet alle Formen einer vielfältigen Demokratie zu bespielen. Die Interkulturanstalten sind so betrachtet nicht nur ein Best-Practice-Beispiel für die Verstetigung einer neo-intermediären Akteurin, sie sind auch Protagonist*innen einer vielfältigen Demokratie. Die Interkulturanstalten sind ein Beispiel dafür, erfolgreich Politik zu machen, indem die unterschiedlichen Ebenen einer vielfältigen Demokratie gleichzeitig nebeneinander bespielt und aufeinander bezogen werden. Das ist nicht zuletzt auch, wenn nicht der, dann zumindest ein umsetzungs- und ergebnisorientierter Weg zur Stärkung der lokalen Demokratie.

\section{Literatur}

Beck, S., Kriemann, M. \& Luchmann, C. (2017): vhw-Studie „Stadtmacherinnen und Stadtmacher". Teil 1: Zwischenergebnisse. Bürgergesellschaft zwischen Government und Urban Governance, vhw-werkSTADT Nr. 11. Berlin.

Beck, S. \& O. Schnur (2016): Mittler, Macher, Protestierer. Intermediäre in der Stadtentwicklung. Berlin.

Gesemann, F., Kriemann, M., Seidel, A., Luchmann, C., Thal, T., Walz, S., Mayer, M., Kammertöns, V. \& Thal, L. (2017): Stadtmacherinnen und Stadtmacher. Zur neuen Vielfalt intermediärer Akteure in Berlin in den Themenfeldern Stadtentwicklung und Flüchtlingspolitik. Abschlussbericht zur Explorationsstudie im Auftrag des Bundesverbands für Wohnen und Stadtentwicklung (vhw). Berlin.

Heinze, R. G. \& Olk, T. (2001): Bürgerengagement in Deutschland - zum Stand der wis-senschaftlichen und politischen Diskussion. In: Heinze, R. G. \& Olk, T. (Hrsg.): Bürgerengagement in Deutschland. Bestandsaufnahme und Perspektiven. Wiesbaden: 11-26.

Interkulturanstalten (2018): „Der Ort“. Abrufbar unter: http://www.interkulturanstalten.de/index.php/ulme-35-raum-fuer-integration-und-kreativitaet/der-ort/. Letzter Zugriff: August 2018 . 
Interkulturanstalten (2017): Zwischenbericht des Vereins Interkulturanstalten Westend e.V., Dezember 2017. Abrufbar unter: http://www.interkulturanstalten.de:60080/a3138ae140 cc91c69d858d12e354ea33226b61db/3eaf4c63-89ae-f4e9-692a-a8126f589586/tap2_O qQOaj_dec/2017_12_05-T_tigkeitsbericht.pdf. Letzter Zugriff: August 2018.

Maikämper, M. (2016): Ākteursmodelle in multilateralen Prozessen der Stadtentwicklung, RaumPlanung 187 (5). 35-41.

Michel, H. (2017): Wir machen das. Mein Jahr als Freiwilliger in einer Unterkunft für Geflüchtete. Köln.

Roth, R. (2015): Intermediäre Akteure in einer „vielfältigen Demokratie“. In: vhw Forum Wohnen und Stadtentwicklung (5), 226-230.

Schiffauer, W., Eilert, A. \& Rudloff, M. (Hrsg.) (2017): So schaffen wir das. Eine Zivilgesellschaft im Aufbruch. 90 wegweisende Projekte mit Geflüchteten, Bielefeld.

Open Access Dieses Kapitel wird unter der Creative Commons Namensnennung 4.0 International Lizenz (http://creativecommons.org/licenses/by/4.0/deed.de) veröffentlicht, welche die Nutzung, Vervielfältigung, Bearbeitung, Verbreitung und Wiedergabe in jeglichem Medium und Format erlaubt, sofern Sie den/die ursprünglichen Autor(en) und die Quelle ordnungsgemäß nennen, einen Link zur Creative Commons Lizenz beifügen und angeben, ob Änderungen vorgenommen wurden.

Die in diesem Kapitel enthaltenen Bilder und sonstiges Drittmaterial unterliegen ebenfalls der genannten Creative Commons Lizenz, sofern sich aus der Abbildungslegende nichts anderes ergibt. Sofern das betreffende Material nicht unter der genannten Creative Commons Lizenz steht und die betreffende Handlung nicht nach gesetzlichen Vorschriften erlaubt ist, ist für die oben aufgeführten Weiterverwendungen des Materials die Einwilligung des jeweiligen Rechteinhabers einzuholen.

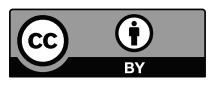

